\title{
The Variability of Stellar Magnetic Flux, Related Surface Structures and Activity
}

\author{
By STEVEN H. SAAR \\ Harvard-Smithsonian Center for Astrophysics, Cambridge, MA 02138, USA
}

I review information from a variety of sources, including magnetic field measurements, Doppler imaging, and various photometric and spectroscopic survey and monitoring programs for clues concerning the physical properties, spatial structure, variability and evolution of magnetic regions and associated activity on cool stars. Based on these data, I sketch a scenario of what the surfaces of cool stars look like as a function of spectral type and age/rotation/activity level.

\section{Introduction}

Recent analyses of long time series of photometry, Ca II fluxes, and absorption line profiles have yielded significant advances in our understanding of stellar magnetic fields, dynamos, surface structures, and their evolution in time. In this review, I briefly summarize these developments, constructing a scenario for how stars of different masses appear and behave as they evolve. To keep things tractable, I will focus on the evolution of cool solar-like $(\mathrm{F}-\mathrm{K})$ dwarf stars from about Pleiades age $\left(t \approx 7 \times 10^{7}\right.$ years $)$ to stars roughly twice the Sun's age $\left(t \approx 9 \times 10^{9}\right.$ years). Stars will be divided into several broad age groups, and the typical magnetic activity characteristics of each group discussed in turn, in order to build up an overall picture of the evolution of magnetic-related phenomena during the life of a cool dwarf star. For a more detailed discussion of some of the issues raised, I refer the reader to Saar \& Baliunas (1992a), Radick (1992) and Saar (1994).

\section{Very young stars (10 $\mathbf{M y r} \leq$ age $\leqslant 0.1 \mathrm{Gyr})$}

Although recent photometry of the Pleiades (Prosser et al. 1993) and other young stars has considerably increased our knowledge of these stars' rotational periods, there is unfortunately little information on either their magnetic properties or their long-term activity variations. Only a few stars this young are nearby enough to have been included in the Mt. Wilson $\mathrm{H}+\mathrm{K}$ programs, and most have photometry spanning a few seasons at best. Some exceptional cases, however, offer vague glimpses of the dynamo properties of these youthful objects.

HD 17925, a K2 dwarf, is thought to be roughly Pleiades age (Cayrel de Strobel \& Cayrel 1989). Detailed models of low and high Zeeman sensitive lines show that for this star, the product of the area filling factor of "bright" magnetic regions (plage/active network), $f_{\mathrm{p}}$, and the mean field strength in these regions $B$, is $f_{\mathrm{p}} B \approx 525 \mathrm{G}$ (Saar 1991a; consistent with Basri \& Marcy 1994). Its Ca II HK record reveals $P_{\text {rot }} \approx 6.8$ days and considerable long-term variability from 1967-present (with an overall downward trend), but no clear cycle (if present, $P_{\text {cyc }} \gtrsim 30$ years). A magnetic flux measurement for the "naked" T Tauri star Tap 35 of $f_{p} B \approx 1000$ G (Basri et al. 1992) with a Li I based age of $t \approx 0.008 \mathrm{Gyr}$, combined with HD 17925, suggest a "saturation" in magnetic flux and $f_{p}$ for $t \lesssim 8.5 \mathrm{Gyr}$ (Saar 1991b; Figure 1). Analysis of $f_{p}$ as a function of rotation (Figure 1) suggests a possible reason for this result: the surface area of plage/network has reached some maximum level. Indeed, if $B$ on Tap 35 shows the pressure equipartition field strengths typical of the cool stars measured to date (e.g., Saar 1991b), it would have $B \sim 1400 \mathrm{G}$, and thus $f_{p} \sim 70 \%$, clearly a significant coverage. 

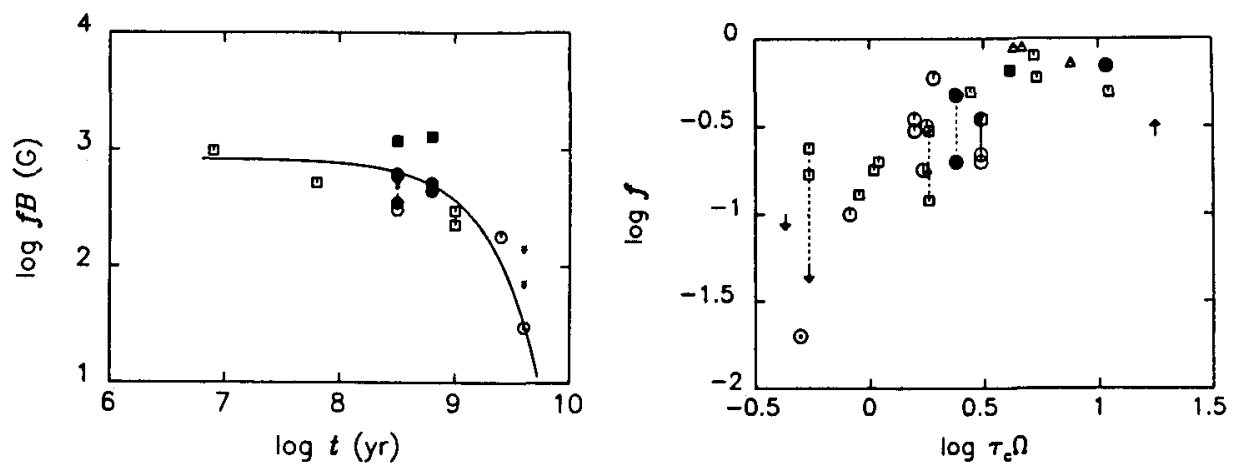

FIgURE 1. (Left): $f B$ vs age $t$. Circles, squares and triangles represent $\mathrm{G}, \mathrm{K}$, and $\mathrm{M}$ stars; filled symbols represent cluster members, open symbols have ages based on $\mathrm{Li}$ I. An exponential fit is shown (solid; $f B \propto e^{-0.82 t}$ with $t$ in Gyr). HD 17925 and Tap 35 define the relation at young ages (from Saar 1991b). (Right): Magnetic filling factor $f$ vs. inverse Rossby number $\tau_{c} / P_{\text {rot }}$, where $\tau_{c}$ is the convective turnover time (e.g., Noyes et al. 1984). Here, open symbols are dwarfs and filled are lower gravity stars; the Sun is denoted by $\odot ; \Omega \equiv P_{\text {rot }}^{-1}$. Different measurements of the same object are connected by dotted lines. $f \propto\left(\tau_{c} \Omega\right)^{\alpha}(1 \leq \alpha \leq 2)$. for low $\tau_{c} \Omega$; saturation sets in above $\tau_{c} / \Omega \gtrsim 4$ (from Saar 1991b).

HD 129333 (G0V; $P_{\text {rot }} \approx 2.8 \mathrm{~d}$ ), one of the most active nearby G dwarfs, is also believed to be of Pleiades age. It displays a generally increasing trend in its $\mathrm{Ca}$ II flux, but no clear cycle (the earliest observations, however, date from 1978 , so only short $P_{\text {cyc }}$ are ruled out). Surface differential rotation (SDR) based on changes in photometric periods, is of order 0.1 of the solar value (E. Guinan 1993, private communication). The star does appear to show an anticorrelation between $\mathrm{V}$ band luminosity and $\mathrm{Ca}$ II flux, in the sense that the star is more active whan it is darker (Radick 1992), with $f_{s} \approx 5 \%$ (Noyes et al. (1991). This trend is opposite of the solar case, but appears to be typical in young stars, and is discussed in more detail in the next section.

LQ. Hya ( $=\mathrm{HD} 82558 ; \mathrm{K} 2 \mathrm{Ve} ; P_{\text {rot }}=1.6 \mathrm{~d}$ ) is also roughly Pleiades age, and one of the most active nearby $\mathrm{K}$ dwarfs. It has recently been Doppler imaged by two groups (Strassmeier et al. 1993; Saar et al. 1992, 1994), and shows an overall spot coverage of $f_{s} \sim 10 \%$. Magnetic images, while only approximate, suggest the presence of significant flux almost everywhere and $f_{p} \approx 50 \%$ if $B=2000 \mathrm{G}$. The images imply that dark spots on LQ Hya generally appear at higher latitudes than on the Sun, with significant spot coverage even near the poles. This picture of "polar" spots is supported by theory. Schüssler \& Solanki (1992) suggest that such polar spots are expected on rapid rotators, where rotational effects dominate magnetic buoyancy and cause flux tubes to rise along equi-velocity surfaces rather than radially. From the study of the latitudinal extents of polar and equatorial spots, Saar et al. (1994) find an upper limit to surface differential rotation (SDR) rate of LQ Hya of $\lesssim 0.003$ of the solar value. Though no long-term Ca II data is available, there is some evidence for a 6.2 year photometric cycle (Jetsu 1993).

Finally, the well-studied BY Dra variable BD $+26^{\circ} 730$ (e.g., Bopp et al. 1983), though a member of the Hyades $(t \approx 0.7 \mathrm{Gyr})$, rotates in just 1.8 days due to tidal synchronization with an unseen spectroscopic companion. It may thus be valid to compare $\mathrm{BD}+26^{\circ} 730$ with other stars in this "very young" group, since it has the $P_{\text {rot }}$ of a Pleiades star one tenth as old. $\mathrm{BD}+26^{\circ} 730$ shows a clear long-term $\left(P_{\mathrm{cyc}} \sim 60\right.$ year $)$ photometric cycle. Magnetic field (Saar et al. 1990) and molecular band analysis (Saar \& Neff 1990) imply that the star has $f_{p} \approx 50 \%$ and $f_{s} \approx 20 \%$. Since the stellar inclination is only 
$i \approx 20^{\circ}$, these results imply large magnetic flux and spot area near the pole. Little change in C IV $1550 \AA$ and $\mathrm{H} \alpha$ fluxes have been observed between 1981 and 1992, despite significant changes in photometric brightness (Saar et al. 1990). A possible explanation lies in the fact that $\mathrm{BD}+26^{\circ} 730$, as well as $\mathrm{HD} 129333$ and $\mathrm{LQ} \mathrm{Hya}$, are among the most active stars of their spectral types, with near-saturated levels of chromospheric and transition region emission (cf. Vilhu 1984). The dynamo production of magnetic flux in these stars can alter the relative amounts of $f_{s}$ and $f_{p}$ (and hence brightness) in time, it may be unable to significantly change the total $f B$ (and hence the overall activity level) since $f_{p}+f_{s} \sim 1$.

Thus, although currently available data are sparse, preliminary indications are that very young stars have nearly saturated outer atmospheric emission, magnetic area filling factors, and perhaps surface magnetic flux. They have $f_{p}$ on the order of $40 \%-50 \%$ (or more) and $f_{s} \sim 5 \%$ to $20 \%$ (possibly larger for $\mathrm{K}$ stars than $\mathrm{G}$ stars), yielding spot-toplage/network area ratios much larger than seen on the Sun. Spots are often found at or near the stellar poles, at least in $\mathrm{K}$ stars. The dynamo may show cycles as viewed in spot area (irradiance), but due to (near-)saturation in the chromosphere, other activity indicators can show a variety of behaviors, ranging from anticorrelations with brightness (HD 129333), to only irregular activity variability with no clear periodicity (BD $\left.+26^{\circ} 730\right)$. Thus, dynamo cycles may or may not be apparent, depending on the diagnostic used to search for them. Surface differential rotation appears to be greatly reduced (by factors of 10 to 100 ) relative to the Sun, while rotational periods (for single stars) span a broad range from $P_{\text {rot }} \approx 1$ to 5 days, with $P_{\text {rot }}$ typically increasing from $\mathrm{F}$ to $\mathrm{K}$ stars $\left(\tau_{\mathrm{c}} / P_{\text {rot }} \approx\right.$ constant).

\section{Young stars (0.3 Gyr $\lesssim$ age $\lesssim \mathbf{0 . 8}$ Gyr)}

With this group, we enter into a range of stellar ages which are reasonably well sampled by the long-term $\mathrm{Ca}$ II and photometric surveys. Considerably more data is therefore available. This particular group also encompasses the Hyades $(t \approx 0.7 \mathrm{Gyr})$ and Ursa Major clusters $(t \approx 0.3 \mathrm{Gyr})$, and thus many group members have well determined ages. Rotation periods at this epoch range from $\sim 3$ days ( $F$ stars) to $\sim 12$ days ( $\mathrm{K}$ stars).

The bulk of the magnetic field data for $G$ and $K$ stars also come from this age range (younger stars often have too large $v \sin i$, older ones have too small $f$ values for accurate measurement). Typically, one finds (Saar 1990) $f_{p} \approx 20 \%$ to $50 \%$. These data are also primarily responsible in helping to establish the empirical relationships between magnetic flux and outer atmospheric emission (e.g., Schrijver et al. 1989). Chromospheric, transition region and coronal fluxes are correlated with $f B$ as $F_{\mathrm{chr}} \propto(f B)^{0.5}, F_{\mathrm{TR}} \propto(f B)^{0.7}$, and $F_{\text {cor }} \propto(f B)^{1.0}$ (Schrijver 1991), consistent with simple models (Montesinos \& Jordan 1993). Few direct spot estimates exist; Campbell \& Cayrel (1984) estimate $f_{s} \approx 3 \%$ on the Hyades supercluster member HD 1835, and photometric variations suggest similar values for other Hyads and UMa cluster stars (Noyes et al. 1991). Thus, the $f_{s} / f_{p}$ ratio $(\sim 0.1)$ is smaller here than for the very young stars $(\sim 0.3)$, but still larger than the solar value: $\left.\left(f_{s} / f_{p}\right)_{\odot} \approx 0.1 \% / 2 \%=0.05\right)$. Photometric variability drops below detection limits for stars hotter than about F7 in the Hyades (Radick et al. 1987), suggesting a more even distribution of spots (Giampapa \& Rosner 1984) or an inhibition of large spot formation (Bünte \& Saar 1993) may occur as $T_{\text {eff }}$ increases.

Many of the basic characteristics and trends tentatively identified above in the very young stars are more clearly evident in this group. Analysis of yearly variability in the combined Mount Wilson Ca II (e.g., Baliunas et al. 1985) and Lowell/Cloudcroft photometry (e.g., Radick 1992) clearly shows that stars of Hyades age and younger show 


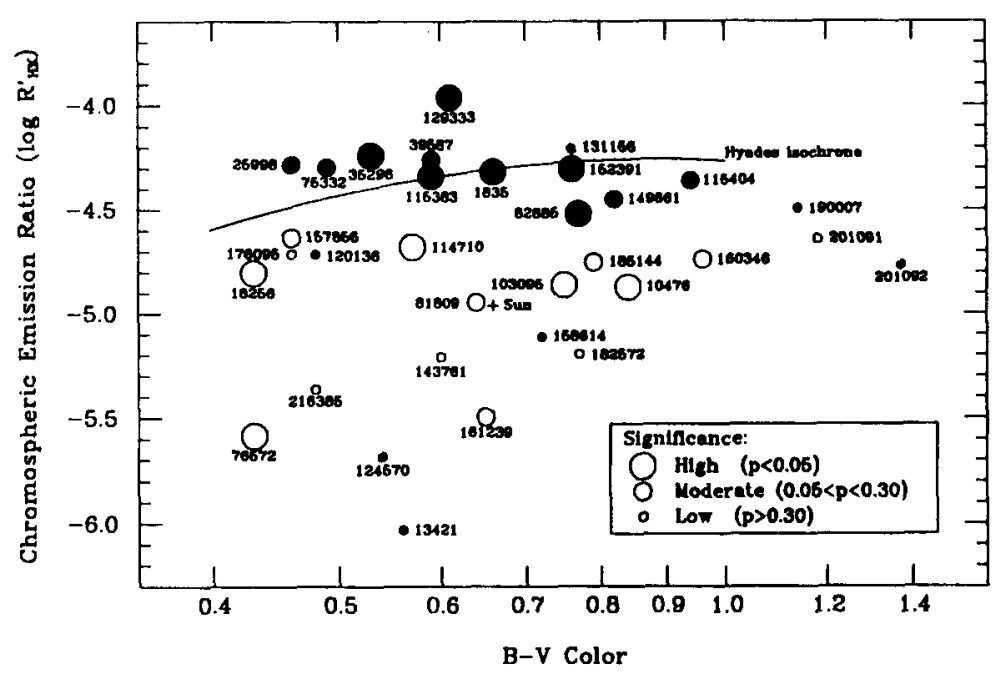

FIGURE 2. Correlation (open symbol = positive, filled $=$ negative) between yearly brightness and $\mathrm{Ca}$ II variations, plotted in a color vs. normalized Ca II flux $R_{\mathrm{HK}}^{\prime}\left(=F_{\mathrm{HK}}^{\prime} / \sigma T_{\text {eff }}^{4}\right)$ diagram (from Radick 1992).

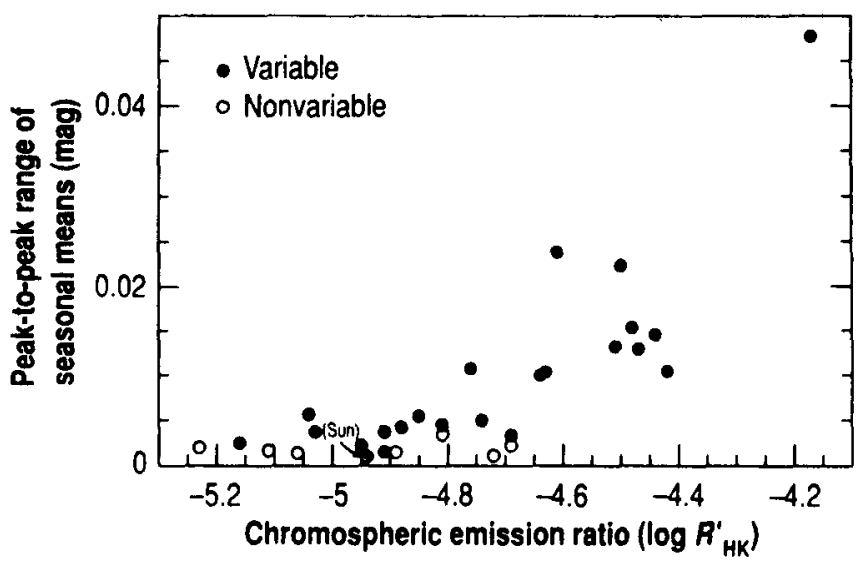

FIGURE 3. Seasonal photometric amplitude vs. $R_{\mathrm{HK}}^{\prime}$ (from Radick et al. 1989).

anticorrelations between brightness and chromospheric activity - stars darken with increased activity (Radick et al. 1987; Figure 2). This fact, combined with evidence that the amplitude of the brightness variations shows a non-linear increase as a function of the $\mathrm{Ca}$ II variations (Figure 3), suggests that dark spots dominate brightness variations in active stars and increase more rapidly than plage with activity (e.g., Radick et al. 1989). These anticorrelations are even seen during the most active solar cycles (Foukal 1993); although more typically, solar plage, network and faculae conspire to overcome the irradiance decrease due to spots and produce a positive correlation between brightness changes and activity (e.g., Foukal \& Lean 1988 and other papers in this volume: e.g., Chapman 1994; Dorren \& Guinan 1994; Fröhlich 1994; Radick 1994).

A casual study of the Mount Wilson Ca II timeseries suggests they may be grouped into several broad morphological classes: irregular variables, cyclic variables (including a 


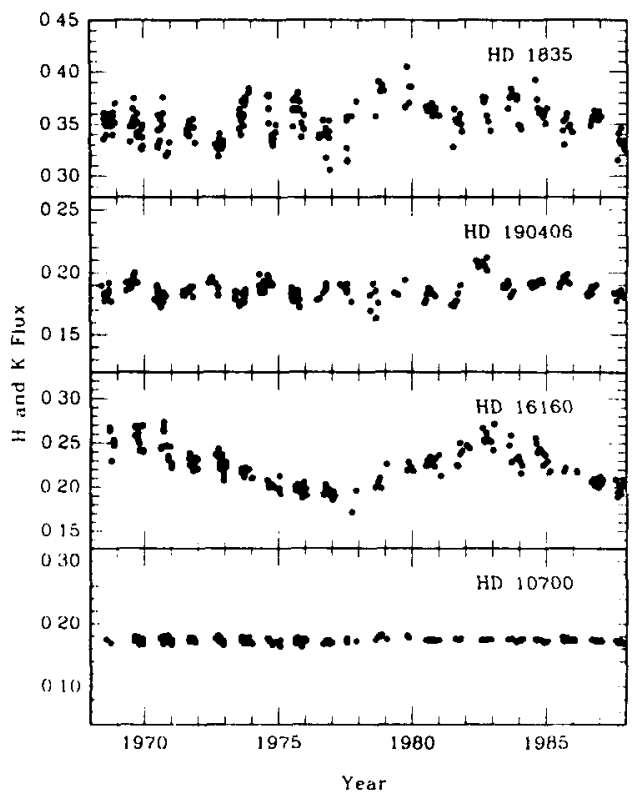

Figure 4. Timeseries of the Mount Wilson Ca II $S$ index for four dwarfs (Baliunas 1991), showing the main types: irregular (HD 1835; $t \approx 0.8 \mathrm{Gyr}$ ), multicyclic (HD 190406; $t \sim 2 \mathrm{Gyr}$ ), cyclic (HD 16160; $t>5 \mathrm{Gyr}$ ), and non-variable (HD 10700; $t>5 \mathrm{Gyr}$ ).

few showing two periods simultaneously), and non-variables (e.g., Wilson 1978; Baliunas \& Vaughan 1985; Figure 4). If a combination of cluster membership and an approximate Ca II flux vs. age relationship (Soderblom et al. 1991; Donahue 1993) is used to approximately date the stars in the sample, we find several general trends. First, there is a general progression from irregular variability, to cyclic and multicyclic behavior, to non-variability, with advancing age (Figure 5). In the age range considered here, most of the stars are irregular variables, although a few cyclic stars exist for $\log t \lesssim 8.6$. The interpretation of this irregular variability is somewhat uncertain. It could be that stars of Hyades/UMa age are still so active that the large changes in $f_{p}$ needed to produce large amplitude $\mathrm{Ca}$ II variations are not possible. Flares and active region growth and decay may be so frequent that these mask the Ca II variations. Perhaps several dynamo "modes" of different amplitude and period $P_{\text {cyc }}$ are operating simultaneously, which when noise is added yields seemingly chaotic timeseries. Throughout, $\mathrm{K}$ stars have larger fractional Ca II variations $A_{\mathrm{HK}} \equiv \Delta F_{\mathrm{HK}} / F_{\mathrm{HK}}^{\prime}$, where $F_{\mathrm{HK}}^{\prime}$ is the Ca II HK flux with the photospheric/basal component removed; see e.g., Noyes et al. 1984) than G stars (Saar \& Baliunas 1992a). Support for the hypothesis that the Ca II cycles are indeed magnetic in origin comes from a four year study of $f B$ and $F_{\mathrm{HK}}^{\prime}$ in $\kappa$ Ceti (Saar \& Baliunas 1992b), in which the two quantities vary in phase during the declining part of a stellar cycle.

More information on the SDR rates is also available from stars in this group. Donahue et al. (1994) compute seasonal values of $P_{\text {rot }}$ from the $S$ dataset, and take the range of these, $\Delta P$, to be a measure (actually a lower limit) to the surface differential rotation rate. Figure 6 shows the resulting $\Delta P$ values plotted against $P_{\text {rot. }}$. A clear correlation is seen, with $\Delta P \propto P_{\text {rot }}^{1.35}$, independent of spectral type (i.e., mass or convective zone depth, $d$ ) on the main sequence. SDR rates for the "young" group are therefore on the order of 0.1 of the solar value (larger in $\mathrm{K}$ stars, smaller in $\mathrm{F}$ stars). Although several selection effects complicate matters, nevertheless, the non-linear relationship and lack of 


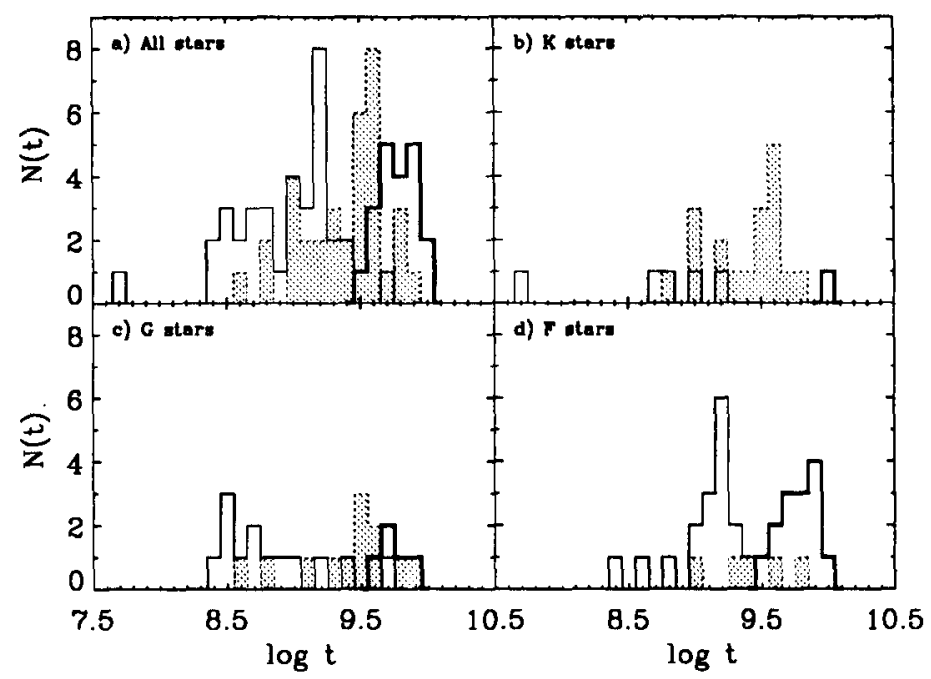

FIGURE 5. Histogram of stellar ages by spectral class and morphology type (thin = irregular, shaded $=$ cyclic $/$ multicyclic, thick=non-variable). Note the trends with spectral type and age (from Saar et al. 1994).

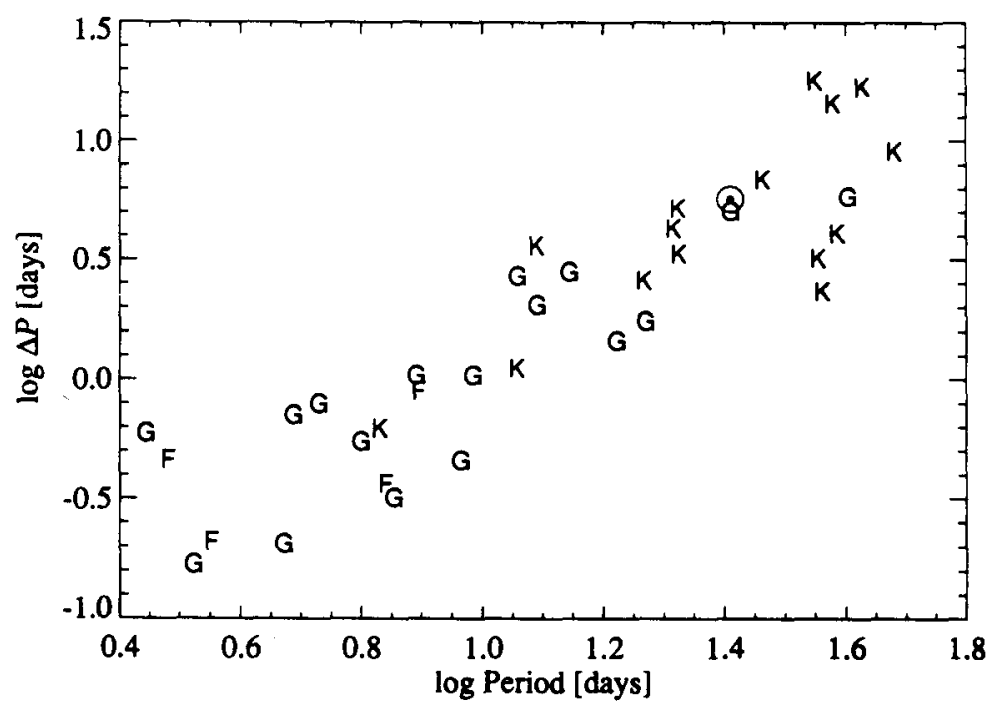

Figure 6. The range of seasonally derived $P_{\text {rot }}$ values, $\Delta P$, vs. $P_{\text {rot }}$ (spectral types as marked; Sun=๑). The best fit yields $\Delta P \propto P_{\text {rot }}^{1.35}$ (Donahue et al. 1994).

spectral type dependence are both at odds with simple theoretical models (e.g., Belvedere et al. 1980; Küker et al. 1993).

Finally, there is evidence that convection is altered on young, active stars. Convective velocities can be estimated from the so-called macroturbulent velocity $\left(v_{\text {mac }}\right)$ derived from detailed models of high resolution absorption line spectra (e.g., Gray 1988). Gray (1984) and Toner \& Gray (1988) already noted enhanced $v_{\text {mac }}$ on active stars, especially $\xi$ Boo A. Saar \& Osten (1994, in preparation) have modeled high resolution ESO coude 


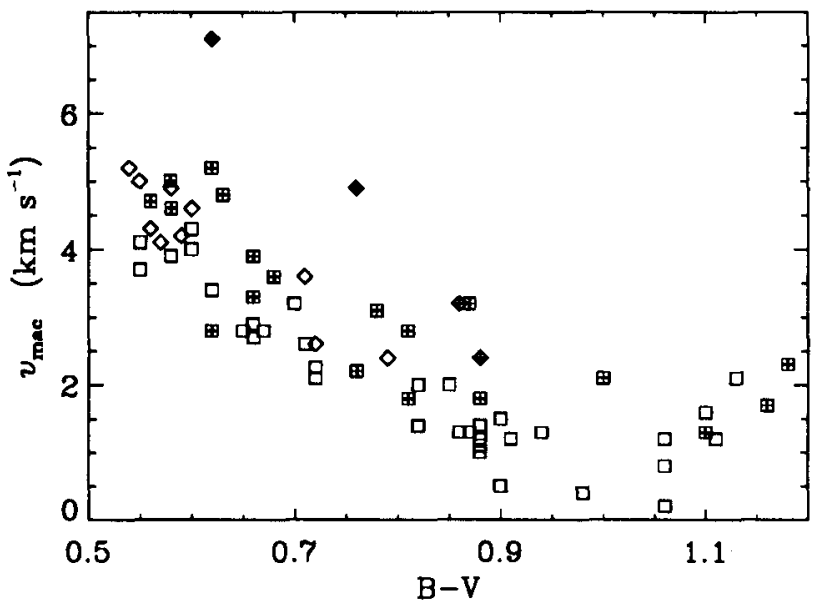

FIGURE 7. Radial-tangential macroturbulence $v_{\text {mac }}$ vs. B-V color; squares are derived from ESO data, diamonds are from Gray (1984), crossed symbols denote stars with significant chromospheric and/or coronal emission (typically with $t \lesssim 2 \mathrm{Gyr}$ ). Note that the mean $v_{\mathrm{mac}}$ for active stars is roughly $1 \mathrm{~km} \mathrm{~s}^{-1}$ more than for inactive stars at fixed color (i.e., fixed $T_{\text {eff }}$ ). From Saar \& Osten (1994, in preparation).

spectra of a large sample of southern dwarfs, and confirm Gray's hypothesis (Figure 7): active stars show $v_{\mathrm{mac}} \approx 1-2 \mathrm{~km} \mathrm{~s}^{-1}$ higher than inactive stars of the same color $\left(T_{\mathrm{eff}}\right)$. Line bisector spans also show variation with stellar cycle (Gray 1992; Livingston 1991). Since convective energy transport is inhibited in magnetic regions (e.g., Livingston 1991), stars where $f$ is large may force convection outside magnetic regions to be that much more violent (e.g., Gray 1992). The enhancement in $v_{\text {mac }}$ is likely present in the very young stars as well, but is difficult to observe due to increased rotational broadening.

\section{Intermediate age stars (age $\approx 1$ to $2 \mathrm{Gyr}$ )}

Intermediate age stars have rotation periods (based on HK data) of 6 days (F stars) $\lesssim P_{\text {cyc }} \lesssim 20$ days (K stars). There are no nearby clusters in this age range, so $t$ must be estimated indirectly (e.g., Soderblom et al. 1991). As $f_{p}$ is declining with $t$ (Figure 1), only a few stars in this group have magnetic measurements. One of the most recent (and accurate) measurements is for the K2 dwarf $\epsilon$ Eri (Valenti \& Basri 1994), which shows $f_{p} \sim 12 \%$ and $B \sim 1900$ G. Dorren \& Guinan (1982) estimate $f_{s}$ of a few percent for several of these stars. For the class as a whole, I estimate $f_{p} \approx 5 \%$ to $20 \%$, and $f_{s} \lesssim 2 \%$, suggesting spot-to-plage ratios only somewhat larger than the Sun. Convective velocities here are subsiding to their "typical" inactive values (Figure 7).

Intermediate age stars are transitional in several respects. Cyclic variability in Ca II becomes more common, especially in $\mathrm{G}$ and $\mathrm{K}$ stars (Figure 5 ). The paucity of cyclic F stars may partly be a threshold problem, however (i.e., $A_{\mathrm{HK}}$ too small to detect). Several of these young cyclic stars are of the multicyclic variety, displaying two distinct $P_{\text {cyc }}$ values. As was the case with the irregular variables, the fractional amplitude $A_{\mathrm{HK}}$ of the cycles increases with decreasing $T_{\text {eff }}$ and increasing $d$ (Saar \& Baliunas 1992a). SDR rates are perhaps a factor of 2 to 3 faster than the Sun (Figure 6) and they can show distinctly non-solar SDR behavior. HD 114710, for example, displays evidence for two activity belts and possibly a reversed pole-to-equator angular velocity gradient 

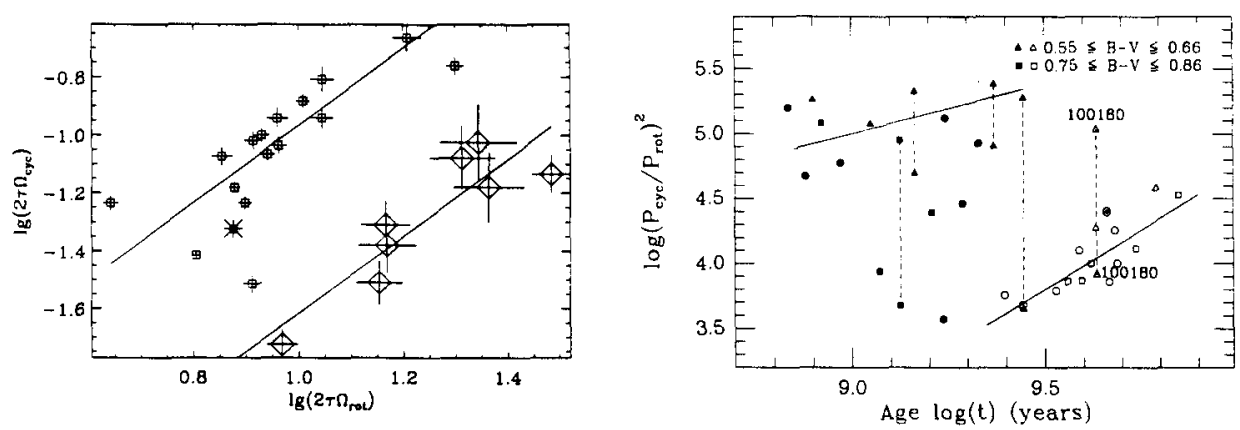

FIGURE 8. Left: Cycle frequency vs. rotational frequency, both normalized to $\tau_{\mathrm{c}}$ (large symbols are young/active, small symbols are old/inactive, the Sun is marked with a $x$ ). All cycles plotted are primary $P_{\text {cyc }}$ values with FAP $\leq 10^{-6}$. Note the segregation of stars into active and inactive branches, each with $\tau_{\mathrm{c}} \Omega_{\mathrm{cyc}} \propto\left(\tau_{\mathrm{c}} \bar{\Omega}_{\mathrm{rot}}\right)^{1.3}$ (solid). Right: $\left(P_{\mathrm{cyc}} / P_{\text {rot }}\right)^{2}$ vs. $t$ (from Soderblom et al. 1991) for stars with a less restrictive FAP $\leq 10^{-2}$ (solid line denotes fits to subsets with FAP $\leq 10^{-5}$ ). Dashed lines connect two $P_{\text {cyc }}$ for multicyclic stars, and filled symbols are active stars. The young/old dichotomy is again apparent, with the transition between 1 and $3 \mathrm{Gyr}$ (from Soon et al. 1993).

(Donahue \& Baliunas 1992). The sense of the brightness-activity relation changes as well, with some G stars showing solar-like positive correlations (e.g., HD 114710; Figure 2) while some $\mathrm{K}$ dwarfs continuing to display young-star-type anticorrelations (e.g., HD 149661; Figure 2). One of the most striking transitional aspects of the intermediate stars however, involves their cycle periods.

Many dynamo theories predict relationships between $P_{\text {cyc }}$ and $P_{\text {rot }}$ (e.g, Robinson \& Durney 1982). Noyes et al. (1984) first noted a relationship between $P_{\text {cyc }}$ and $P_{\text {rot }}$ for inactive, older stars (see next section). Saar \& Baliunas (1992a) studied this issue again, with the benefit of almost 10 more years of data (Baliunas et al. 1994, in preparation). They demonstrated that both inactive and active stars show clear and similar correlations when the best determined $P_{\text {cyc }}$ are used (those with "false alarm probabilities" or FAP $\leq 10^{-6}$ ), and when $P_{\text {cyc }}$ and $P_{\text {rot }}$ are properly normalized (they used the magnetic diffusion timescale and $\tau_{c}$ in two examples). If these steps are taken, the inactive and active stars are displaced into nearly parallel branchs, with inactive stars showing lower $P_{\text {cyc }}$ values (Figure 8 ). If $\tau_{c}$ is used in the normalization, $\tau_{\mathrm{c}} \Omega_{\mathrm{cyc}} \propto\left(\tau_{\mathrm{c}} \Omega_{\mathrm{rot}}\right)^{1.3}$. Note that then (approximately), $\Omega_{\text {cyc }} \propto(\Delta P)^{-1}$, suggesting a possible connection between SDR and the cycle period. Since $\tau_{c} / P_{\text {rot }}$ is, to first order, correlated with stellar age, a transition $P_{\text {cyc }} / P_{\text {rot }}$ is implied at some $t$. Several of the multicyclic stars have a $P_{\text {cyc }}$ value in each regime, suggesting they may represent a transitional phase in evolution between branches. Soon et al. (1993) confirmed this idea using a somewhat different parameterization (Figure 8), and showed that the transition takes place between 1 and 3 Gyr. Perhaps some critical change in the dynamo mechanism (e.g., Knobloch et al. 1981) occurs at this time. Soon et al. (1993) also show that $P_{\text {cyc }} / P_{\text {rot }}$ decreases with $T_{\text {eff }}$ (and d) in both branches.

\section{Old stars (age $\gtrsim 3$ Gyr)}

This group encompasses roughly solar age and older stars, and consistent with this fact, most behavior observed is truly "solar-like". I discuss stars with $t \lesssim t_{\odot}$ first. Typical $P_{\text {rot }}$ values range from 15 days (late $\mathrm{F}$ stars) to 40 days (K stars). Of this class, only the 


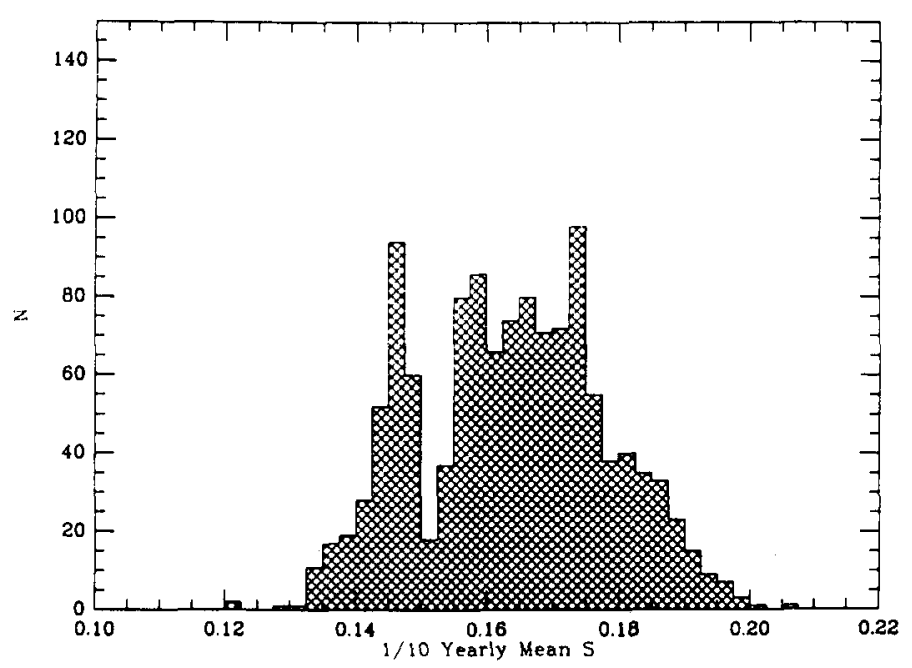

Figure 9. Frequency distribution of the $S$ index ( 0.1 year averages) for inactive $(0.13 \leq\langle S\rangle \leq$ $0.20)$ solar-like $(0.60 \leq(\mathrm{B}-\mathrm{V}) \leq 0.76)$ dwarfs (from Baliunas \& Jastrow 1990).

Sun has reasonably accurate estimates of spot and plage area: $f_{p} \sim 2 \%$ (Montesinos \& Jordan 1993) and $f_{s} \sim 0.1 \%$ SDR rates are similar to the Sun, though proportionately larger in $\mathrm{K}$ stars and smaller in $\mathrm{F}$ stars. Very few irregular Ca II variables remain by this epoch; cycles dominate in the $\mathrm{K}$ stars, while a "new" class, the non-variables, becomes important in the $G$ and especially the $F$ stars (Figure 5). Well determined correlations between brightness and $\mathrm{Ca}$ II variations are almost invariably positive in sign (solar-like), indicating the dominance of plage and network in the irradiance variations. The cyclic variables are found only on low $P_{\text {cyc }}$ branch in the $P_{\text {cyc }}-P_{\text {rot }}$ diagrams (Figure 8).

The non-variable stars deserve special attention, since they have characteristics similar to what we infer the Sun was like during the Maunder and similar "magnetic minima". These are epochs when it is thought that cycles, but not all magnetic activity, temporarily cease. Baliunas \& Jastrow (1990) compiled $S$ values for 74 dwarfs, similar to the Sun in color and age, from the combined Wilson and solar neighborhood surveys. After averaging the data into 0.1 year bins, they found that a histogram of the results showed a sharply bimodal distribution (Figure 9): a narrowly peaked distribution for $S<0.15$, separated by a sharp gap from a broad distribution produced by cyclic variables. Stars below the gap have no cycles, and lie at the minimum $S$ levels for their spectral types. Baliunas \& Jastrow (1990) suggest that the low activity, non-varying stars in the low $S$ group are, in fact, experiencing the stellar equivalent of the Maunder Minimum, noting that the fraction of stars in this group is similar to the fraction of time the Sun spends in a quiescent magnetic state. If this hypothesis is correct, and the non-variable stars are in Maunder-like states, several statements can be made: 1) The frequency and/or duration of Maunder-type minima drops sharply with increasing $d$ (Figure 5). 2) The Maunder phenomenon begins rather abruptly at $\log t \approx 9.5$. 3) For stars younger than this, it is possible to have cyclic dynamos without Maunder-type minima. This suggests the possibility that Maunder-like states increase in frequency and/or duration with $t$, though there is little direct evidence for this.

In stars older than the Sun, the F dwarfs will have mostly evolved off the main sequence. For the $\mathrm{G}$ and $\mathrm{K}$ stars, an extrapolation of the above trends suggests $P_{\text {rot }}$ will range from 
30 to 50 days, SDR will increase above solar values, $f_{p}$ and $f_{s}$ will decrease further, and cyclic variability and Maunder minima will dominate the $\mathrm{Ca}$ II timeseries morphology (with $\mathrm{K}$ stars more often showing cycles and $\mathrm{G}$ stars Maunder minima). $P_{\text {cyc }} / P_{\text {rot }}$ should increase with time along the inactive tracks in Figure 8 . There is no concrete evidence that the dynamo ever completely "shuts off".

Acknowledgments. I gratefully acknowledge preprints from and useful and informative discussions with many of those referenced below! Special thanks are due S. Baliunas, A. Brandenburg, R. Donahue, and E. Guinan for permission to discuss unpublished results and to R. Osten for help with the references. I thank ESO and NOAO for generous allocations of telescope time.

\section{REFERENCES}

BALIUNAS S.L. 1991 The past, present, and future of solar magnetism: stellar magnetic activity. In The Sun in Time (ed. M. Matthews, C.P. Sonnett \& M.S. Giampapa). pp. 809-831. Univ. Arizona Press, Tucson, AZ, USA.

Baliunas, S.L. \& Vaughan, A.H. 1985 Stellar activity cycles. Ann. Rev. Astron. Astrophys. 23, 379-412.

Baliunas, S.L. \& Jastrow, R. 1990 Evidence for long-term brightness changes of solar-type stars. Nature 348, 520-523.

Baliunas, S.L., Horne, J.H., Porter, A., Duncan, D.K., Frazer, J., Lanning, H., Misch, A., Mueller, J., Noyes, R.W., Soyumer, D., Vaughan, A.H. \& Woodard, L. 1985 Time-series measurements of chromospheric $\mathrm{Ca}$ II $\mathrm{H}$ and $\mathrm{K}$ emission in cool stars and the search for differential rotation. Astrophys. J. 294, 310-325.

BASRI, G.S. \& MARCY, G.W. 1994 Zeeman enhancement of lines in extremely active K dwarfs. Astrophys. $J$, in press.

BASRI, G.S., MarcY, G.W. \& VAlentI, J. 1992 Limits on the magnetic flux of pre-mainsequence stars. Astrophys. J. 390, 622-633.

Belvedere, G., Paterno, L. \& Stix, M. 1980 Differential rotation along the lower main sequence: A theoretical investigation. Astron. Astrophys. 88, 240-247.

Bopp, B.W., Africano, J.L., Stencel, R.E., Noah, P.V. \& Klimke, A. 1983 Observations of active chromosphere stars. Astrophys. J. 275, 691-703.

BüNTE, M. \& SAAR, S. H. 1994 The interchange instability in stellar magnetic flux tubes. Astron. Astrophys. 271, 167-174.

Campbell, B. \& CaYrel, R. 1984 Spectroscopic evidence for starspots in the G dwarf HD 1835. Astrophys. J. 283, L17-L20.

Cayrel de Strobel, G. \& Cayrel, R. 1989 Strong lithium in the very nearby $\mathrm{K}$ dwarf HD 17925. Astron. Astrophys. 218, L9-L11.

Chapman, G.A. Photometric observations of the Sun. In The Sun as a Variable Star: Solar and Stellar Irradiance Variations (ed. J.M. Pap, C. Fröhlich, H.S. Hudson \& S.K. Solanki). Cambridge Univ. Press, in press.

Dorren J.D. \& Guinan, E.F. 1982 Evidence for starspots on single solar-like stars. Astron. J. 87, 1546-1557.

Donahue, R.A. $1993 \mathrm{PhD}$ thesis, New Mex. State Univ.

Donahue, R.A.\& Baliunas, S.L. 1992 Evidence of differential surface rotation in the solartype star HD 114710. Astrophys. J. 393, L63-66.

Donahue, R.A., SaAR, S.H. \& Baliunas, S.L. 1994 Differential rotation in cool dwarfs: A power law relationship between mean rotation period and its observed range. Astrophys. $J .$, submitted.

Dorren, J.D. \& Guinan, E.F. 1994 The Sun in time: Detecting and modelling magnetic 
inhomogenities on solar-type stars. In The Sun as a Variable Star: Solar and Stellar Irradiance Variations (ed. J.M. Pap, H.S. Hudson, C. Fröhlich \& S.K. Solanki). Cambridge Univ. Press, in press.

Foukal, P. 1993 The curious case of the Greenwich faculae. Solar Phys. 148, 219-232.

FouKaL, P. \& LEAN, J. 1988 Magnetic modulation of solar luminosity by photospheric activity. Astrophys. J. 328, 347-357.

FröHLICH, C. 1994 Irradiance observations of the Sun. In The Sun as a Variable Star: Solar and Stellar Irradiance Variations (ed. J.M. Pap, C. Fröhlich, H.S. Hudson \& S.K. Solanki). Cambridge Univ. Press, in press.

Giampapa, M.S. \& RosNer, R. 1984 The appearance of magnetic flux on the surfaces of the early main-sequence F stars. Astrophys. J. 286, L19-L22.

GraY, D.F. 1984 Measurements of rotation and turbulence in F, G, and K stars. Astrophys. $J$. 281, 719-722.

Gray, D.F. 1988 Lectures on Spectral Line Analysis: F, G, and K Stars (Arva).

GraY, D.F. 1992 Stellar convection: the observations. In Cool Stars, Stellar Systems, and the Sun (ed. M. Giampapa \& J. Bookbinder). ASP Vol. 26, 127-138.

Jetsu, L. 1993 A decade of photometry of LQ Hydrae. Astron. Astrophys. 276, 345-352.

KNoBloch, E., Rosner, R. \& WeIss, N.O. 1981 Magnetic fields in late-type stars. Mon. Not. R. A. S. 197, 45P-49P.

KüKER, M., RÜDIGER, G. \& KrTchatinov, L.L. 1993 An $\alpha \Omega$ model of the solar differential rotation. Astron. Astrophys. 279, L1-L4.

LIVINGSTON, W. 1991 Sun as a star: its convective signature and the activity cycle. In IAU Colloq. 130, The Sun and Cool Stars: Activity, Magnetism, Dynamos (ed. I. Tuominen, D. Moss \& G. Rüdiger). pp. 246-251. Springer.

Montesinos, B.M. \& Jordan, C. 1993 On magnetic fields, stellar coronae, and dynamo action in late-type dwarfs. Mon. Not. R. A. S. 264, 900-918.

Noyes, R.W., Hartmann, L.W., Baliunas, S.L., Duncan, D.K. \& Vaughan, A.H. 1984 Rotation, convection, and magnetic activity in lower main-sequence stars. Astrophys. $J$. 279, 763-777.

Noyes, R.W., Weiss, N.O. \& VAUghaN, A.H. 1984 The relation between stellar rotation rate and activity cycle periods. Astrophys. J. 287, 769-773.

Noyes, R.W., Baliunas, S.L. \& Guinan, E.F. 1991 What can other stars tell us about the Sun? In The Solar Interior and Atmosphere (ed. A.N. Cox, W.C. Livingston \& M.S. Matthews). pp. 1161-1185. Arizona Univ. Press, Tucson, AZ, USA.

Prosser, C.F., Shetrone, M.D., Marilli, E., Catalano, S., Williams, S.D., Backman, D.E., Laaksonen, B.D., Adige, V., Marschall, L.A. \& Stauffer, J.R. 1993 Rotation periods of open cluster stars II. Publ. Astron. Soc. Pac. 105, 1407-1414.

RADICK, R.R. 1992 Luminosity variability of lower main-sequence stars. In The Solar Cycle (ed. K. Harvey). ASP Vol. 27, 450-464.

RADICK, R.R. 1994 Photometric variations of solar type stars. In The Sun as a Variable Star: Solar and Stellar Irradiance Variations (ed. J.M. Pap, C. Fröhlich, H.S. Hudson \& S.K. Solanki). Cambridge Univ. Press, in press.

Radick, R.R., Thompson, D.T., Lockwood, G.W., Duncan, D.K. \& Baggett, W.E. 1987 The activity, variability, and rotation of lower main-sequence Hyades stars. Astrophys. $J$. 321, 459-472.

Radick, R.R., LockWood, G.W. \& Baliunas, S.L. 1989 Stellar activity and brightness variations: A glimpse at the Sun's history. Science 247, 39-44.

Robinson, R.D. \& DURNeY, B.R. 1982 On the generation of magnetic fields in late-type stars: a local time-dependent dynamo model. Astron. Astrophys. 108, 322-325.

SAAR, S.H. 1990 Magnetic fields on solar-like stars: The first decade. In IAU Symposium 138, The Solar Photosphere: Structure, Convection, and Magnetic Fields (ed. J. O. Stenflo). pp. 427-441. Kluwer. 
SAAR, S.H. 1991a Recent advances in the observation and analysis of stellar magnetic fields. In IAU Colloquium 130, The Sun and Cool Stars: Activity, Magnetism, Dynamos (ed. I. Tuominen, D. Moss \& G. Rüdiger). pp. 389-401. Springer.

SAAR, S.H. 1991b Recent measurements of stellar magnetic fields. In Mechanisms of Chromospheric and Coronal Heating (ed. P. Ulmschneider, E. Priest \& R. Rosner). pp. 273-278. Springer.

SAAR, S.H. 1994 The time evolution of magnetic flux, dynamos, and surface structures on cool stars. In The Eighth Cambridge Workshop on Cool Stars, Stellar Systems, and the Sun (ed. J.-P. Caillault). ASP, in press.

SAAR, S.H. \& NEFF, J.E. 1990 Spot temperatures and area coverages on active dwarf stars. In Cool Stars, Stellar Systems, and the Sun (ed. G. Wallerstein). ASP Vol. 9, 171-173.

SAAR, S.H. \& Baliunas, S.L. 1992a, Recent advances in stellar cycle research. In The Solar Cycle (ed. K. Harvey). ASP Vol. 27, 150-167.

SAaR, S.H. \& Baliunas, S.L. 1992b The magnetic cycle of Kappa Ceti (G5V). Ibid, 197-202

SAAR, S.H., Golub, L., Bopp, B.W., Herbst, W. \& Huovelin, J. 1990 Long and short timescale variability of magnetic activity on the BY Dra star BD $+26^{\circ} 730$. In Evolution in Astrophysics: IUE Astronomy in the Era of New Space Missions (ed. E. Rolfe). ESA SP-310, 431-434.

SAAR, S.H., Piskunov, N.E. \& TUOMINen, I. 1992 Magnetic surface images of the BY Dra Star HD 82558. In The Seventh Cambridge Workshop on Cool Stars, Stellar Systems, and the Sun (ed. M.S. Giampapa \& J.A. Bookbinder). ASP Vol. 26, 252-255.

SaAR, S.H., Piskunov, N.E. \& TuOMinen, I. 1994a Multiepoch magnetic surface images of LQ Hya In The Eighth Cambridge Workshop on Cool Stars, Stellar Systems, and the Sun (ed. J.-P. Caillault). ASP, in press.

SaAR, S.H., Brandenburg, A., Donahue, R.A. \& Baliunas, S.L. 1994b The evolution of stellar dynamo variations. In The Eighth Cambridge Workshop on Cool Stars, Stellar Systems, and the Sun (ed. J.-P. Caillault). ASP, in press.

SCHRIJver, C.J. 1991 Relations between magnetic fields and activity. In Mechanisms of Chromospheric and Coronal Heating (ed. P. Ulmschneider, E. Priest \& R. Rosner). pp. 257-272. Springer.

SchriJver, C.J., Coté, J., ZwaAN, C. \& SAAR, S.H. 1989 Relations between the photospheric magnetic field and the emission from the outer atmospheres of cool stars. I. The solar Ca II K line core emission. Astrophys. J. 337, 964-976.

Schüssler, M. \& Solanki, S.K. 1992 Why rapid rotators have polar spots. Astron. Astrophys. 264, L13-L16.

Soderblom, D.R., Duncan, D.K. \& Johnson, D.H.R. 1991 The chromospheric emission-age relation for stars of the lower main sequence and its implications for the star formation rate. Astrophys. J. 375, 722-739.

Soon, W.H., Baliunas, S.L. \& ZHANG, Q. 1993 An interpretation of cycle periods of stellar chromospheric activity. Astrophys. J. 414, L33-L36.

Strassmeier, K., Rice, J.B., Wehlad, W.H., Hill, G.M. \& Matthews, J.M. 1993 Surface features in the lower atmosphere of HD 82558 = LQ Hya. Astron. Astrophys. 268, 671-683.

Toner, C.G. \& GraY D.F. 1988 The starpatch on the G8 dwarf $\xi$ Bootis A. Astrophys. J. 334, 1008-1020.

VAlenti, J. \& BASRI, G. 1994 Multiline Zeeman analysis of $\epsilon$ Eri in the near infrared. In The Eighth Cambridge Workshop on Cool Stars, Stellar Systems, and the Sun (ed. J.-P. Caillault). ASP, in press.

VILHU, O. 1984 The nature of magnetic activity in lower main sequence stars. Astron. Astrophys. 133, $117-126$.

WILson, O.C. 1978 Chromospheric variations in main sequence stars. Astrophys. J. 266, 379396. 